$\begin{array}{llllll}\text { I } & \mathbf{N} & \mathbf{H} & \mathbf{A} & \mathbf{I} & \mathrm{T} \text {. }\end{array}$

Zueignung. - $\quad$ - $\quad$ - $\quad$ Scite 1

Der verschünte Beruf, von Friedr. von Klotz. - 3

Der Morgen, bei einem Spatziergange an der Mosel, Epistel an einen Freund, von IIeinrich von Criegern. $\quad-\quad$ - $\quad-\quad-\quad-6$

Abendempfindung, von E. Teke. - - 8

Alnung, von demselben. - - - $\quad$ - $\quad$ - 9

Die Vermählungsfeier des Rheins mit der Mosel, von Friedrich v. Klotz. - - - 10

Das Veilchen, von Fr. Zimmermann. $\quad-12$

Der Knabo und das Eccho, von Emil Rei-

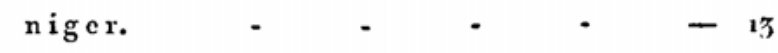

T.uschung, von G. Sze. $\quad-\quad-\quad-15$ 
Preis der Dichtkunst, von Fricdr. v. Flotz. Seite 16

Die Freundschaft, von Fr. Zimmermann. - 18

Des Freundes Schattenrifs, von Heinrich v.

Criegern. $\quad-\quad$ - $\quad-\quad-\quad-23$

Lied der Hoffnung eines Braminen, von 0 .

Ferber. - - $\quad$ - $\quad$ - $\quad$ - $\quad-26$

Elegie, von G. Sze. - - - 27

Früllingsnähe, von Fr. Leben. - - 29

Das Blümchen, von Emil Reiniger. - 31

Kriegerschicksal, von Friedr. von Klotz. - 34

Kriegerweihe, von demselben. - - $\quad 55$

Schützenlied, von Fr. L eben. - - $\quad 36$

Das Ideal, von H. von $\mathrm{L}$ angen. - - 58

Emma, von G. Sze. - - - $\quad$ - 40

Sonett, von demselben. - - - $\quad$ - 41

Sehnsucht nach der Heimath, von H. von

Langen. $\quad-\quad-\quad-\quad-\quad-42$

Verlorner Friede, von Fr. von Flem ming. - 45

Trost, von A. Martini. - - - $\quad 47$

Kriegers Liebe, von Fr. Leben. - - 49

Filage, von II. von $\mathrm{L}$ angen. - - 50

Der Völkerkrieg, von Friedr. v. Klotz. - 51

Bei der Nachricht von der Einnalıme von Paris,

von Heinrich von Criegern. - $\quad$ - 5 ; 
Rannerlieder, von H. Bernhardi:

1. Klage um die Ertrunknen. - Seite 56

2. Ansicht vom Melibokus. - $\quad 58$

3. Der letzte Abend vor Mainz. - - 60

4. Landleben. $\quad-\quad$ - $\quad-\quad-62$

5. Die Heimkehr. $\quad-\quad-\quad-64$

Das Glück des Sängers, von Emil Reiniger. - 66

Syrinx, von Fr. Zimmermann. - - 68

Der Krieger, von Fmil Reiniger. - - 71

Das reine Leben, von Heinrich von Criegern. - 73

Gesang, von G. Sze. - - - - 78

Bescheidne Liebe, von $H$. von Langen. - $\quad 79$

Romanze, von G. Sze. - - - - $\quad$ - 80

Untreue, von Emil Reiniger. - - $8 x$

An Louisen, Madrigal von H. von Langen. -83

Selunsucht, von Fr. Leben. - - 84

Die Nachtigallen, von $H$. von $L$ angen. $\quad-85$

Die Erfüllung, von Emil feiniger. $\quad-\quad 37$

Erhebung, von A. Heinze. - - 89

Wechselgespräch des Dichters mit der Zeit, von

IIeinrich von $\mathrm{Criegern.} \mathrm{-} \quad-9^{\mathbf{1}}$

Der teutsche Mann, von Friedr. von Klotz. - $9^{6}$

Die Geisterschlacht, von H. Bernhardi. - 97

Trostsprïchwort, ron Fricdr, von Filotz. - 102 
Am Rhein, von G. Sze. - - Seite 104

Nierensteiner, von Bornh ardi. - - 106

Trinklied an der Mosel, von Fr. von Klotz. - 109

Das Rheingau, von G. Sze. - - 111

Der Bacchusdiener an scinen Gott, von Heinrich

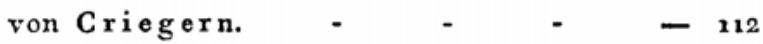

Des Teutschen Wiederkehr, von Emil Reiniger. - 114

Die Ruinen am Rluein, von G. Sze. - 116

Schwermuth, am Rheine, von Fr. Leben. - 117

Sonette, von $H$. von $L$ angen :

1. An Rosa. $\quad$ - $\quad$ - $\quad$ - $\quad$ - 118

2. Sehnsucht. - $\quad-\quad$ - $\quad-119$

3. An Louisen. - $\quad$ - $\quad-121$

4. Zu ihr! - - - - $\quad-122$

An die Geliebte, von Fr. Leben. - - 123

Cliristmette, von G. Sze. - - - - $\quad$ - 224

An Ihrem Namenstage, dem Charfreitage, von

H. von $L$ angen. - - - $\quad-126$

Teutsche Frauenwürde, von Friedr. v. Klotz. - 128

Romanze, von G. Sze. $\quad-\quad$ - $\quad-129$

Liebesklage, von $\mathrm{H}$. von $\mathrm{L}$ angen. $\quad$ 13t

Guido, von O. Ferber. - - - - 132

Was ich verlor und was mir blieb, von Fr.

von Flemming. $\quad-\quad-\quad-134$ 
Die drei Blumen, von Ed. Teke.

Seite 136

Die Realisation, von II. von L a ngen. - 137

Des Mädchens Stimme, von Ed. Teke. - 158

Meine Mädchen, von Emil Reiniger. $\quad-\quad 239$

Venus Aphrodite, von Fr. Zimmermann. - $14 \mathrm{r}$

Der Schiffer, von G. Sze. - - - - 143

Die Thränen, von Fr. Zimmermann. - 144

Erweckung, von A. Kummer. - - 147

Grufs an Freund $p^{* *}$, bei seinem Eintritt in den Banner der freiwilligen Sachsen, von Heinrich von Criegern. $\quad-\quad-\quad-148$

Germania, von A. Kummer. - - 150

Wahlspruch, von Friedr. von Klotz. $\quad 152$

Lied, von Fr. Leben. - - - $\quad$ - 152

Die Jahreszeiten, von Emil Reiniger. - 154

Sehnsucht, von Ed. Teke. - - - - 155

Trinklied, von Emil Reiniger. - - $\quad-157$

Lieschen, von demselben. - $\quad 159$

Die Blumen, von G. Sze. - - - - $\quad-12$

Die Liebe, von demselben. - - 163

Die Kunst, von Fr. Zimmermann. - $\mathbf{1 6}$.

Phantasic, bei einer Hörnermusik auf der Fel. senspitze bey Alten-Alr, von Fr. Leben. $\quad-165$ 


\section{Sonette, von *}

1. Prinz Ludwig von Preufsen, Seite 169

2. Saragossa. - - - $\quad$ - 7 ,

3. Moskau. - - 171

4. Der Rheinūbergang bei Laub. $\quad-172$

Blicke in die Zukunft, von Friedr. von Klotz. - 175

Anmerkungen und Erläuterungen. $\quad-175$ 


\section{LIEDER A US DER FREM DE.}


